\title{
Cave Laborbudget in fachgleichen Gemeinschaftspraxen
}

Auch nach der Laborreform zum 1.10.2008 gilt weiterhin, dass für die veranlassten wie auch selbst erbrachten kurativ-ambulanten Laborleistungen je Vertragsarzt ein sogenanntes Laborbudget gebildet wird. Zudem wird jedem Vertragsarzt der in Kap. 32.1 EBM genannten Fachgruppen je kurativ-ambulantem Fall von seiner Kassenärztlichen Vereinigung (KV) automatisch ein Bonus für die wirtschaftliche Erbringung bzw. Veranlassung von Laborleistungen gewährt (Nr. 32001 EBM). Ausgenommen sind nur Überweisungsfälle mit Auftragsleistung.

Bei Überschreiten des Laborbudgets wird der Überschreitungsbetrag von der Summe der zuerkannten Wirtschaftlichkeitsboni abgezogen. Der EBM regelt eindeutig, dass dieser Abgleich je Vertragsarzt erfolgt. Auch in fachgleichen Berufsausübungsgemeinschaften (Gemeinschaftspraxen) besteht somit keine Möglichkeit der Verrechung des Laborbudgets sowie des Wirtschaftlichkeitsbonus untereinander. Sofern ein vertragsärztliches Mitglied einer solchen Gemeinschaftspraxis deshalb seinen Budgetrahmen überschreitet und somit keinen oder keinen vollständigen Wirtschaftlichkeitsbonus erhält, kann die Überschreitung nicht mit dem eventuell noch vorhandenen Laborbudget seines Kollegen verrechnet werden.

Praxistipp: Obwohl die Verrechnungsmöglichkeit bei der Betrachtung je Vertragsarzt nicht gegeben ist, bringt diese Regelung auch Vorteile für die fachgleichen Gemeinschaftspraxen. Seit Einführung des Vertragsarztrechtsänderungsgesetzes zum 3. Quartal 2008 werden die Leistungen in der Quartalsabrech- nung mit der lebenslangen Arztnummer (LANR) gekennzeichnet. Wird ein Patient in einem Quartal von zwei Ärzten einer solchen Praxis behandelt, wird auch der Wirtschaftlichkeitsbonus für beide Ärzte gewährt. Voraussetzung ist allerdings, dass die Behandlung jeweils durch die Kennzeichnung einer Leistung mit der jeweiligen LANR erfolgt. Da dieser Wirtschaftlichkeitsbonus außerhalb der Regelleistungsvolumina und $\mathrm{zu}$ einem festen Punktwert vergütet wird, entsteht in einer Gemeinschaftspraxis im Gegensatz zu früher ein bedeutungsvoller Honoraranteil. Dieser kann lediglich durch den o.g. Mechanismus gemindert werden. Es kommt deshalb gerade hier ganz besonders darauf an, dass die Kennzeichnung von Fällen mit der LANR exakt zugeordnet ist. Da in einer Gemeinschaftspraxis die einzelnen Partner meist zu gleichen Teilen arbeiten, sollte deshalb eine asymmetrische Verteilung der selbst erbrachten bzw. veranlassten Laborleistungen und damit eine Minderung des Wirtschaftlichkeitsbonus selten vorkommen.

In diabetologischen Schwerpunktpraxen kommt noch hinzu: Bei einer dort oft möglichen Kennzeichnung des Falles mit der Laborbudgetausnahme-Ziffer 32022 EBM (manifester Diabetes mellitus) ist eine Anrechnung der erbrachten und veranlassten Laborleistungen auf das Laborbudget und damit eine Anrechnung auf das Honorar aus dem Ansatz des Wirtschaftlichkeitsbonus nach Nr. 32001 EBM ausgeschlossen.

\section{Präventionslabor: eine Änderung der Änderung beachten!}

Ein Beschluss des Bewertungsausschusses sah ab dem 1.10.2009 vor, dass die Gesundheitsuntersuchung nach Nr. 01732 EBM und die Untersuchung auf Blut im Stuhl nach Nr. 01734 EBM um die darin enthaltenen Laborparameter bereinigt wird. Nun ist er erneut geändert worden. Da die dort enthaltene Urinuntersuchung üblicherweise im Eigenlabor der Praxis erfolgt, ist die Komplexleistung nach Nr. 32890 EBM in die Einzelleistungen 32880 EBM (Urinuntersuchung), 32881 EBM (Glukosebestimmung) und 32882 EBM (Cholesterinbestimmung) aufgeteilt worden. Darüber hinaus wurde die Laborpauschale nach Nr. 32892 für die Untersuchung auf Blut im Stuhl wieder abgeschafft und in die Nr. 01734 EBM zurückgeführt.

Praxistipp: Die Leistungen werden extrabudgetär vergütet und können entweder direkt in der Praxis erbracht oder an die Laborgemeinschaft im Rahmen der Direktabrechnung delegiert werden. Hierzu müssen bis zu einer Formularanpassung auf der Überweisung nach Muster 10A das Ankreuzfeld „präventiv“ markiert und die Parameter Cholesterin (Feld 19) für die Nr. 32882, Glukose (Feld 28) für die Nr. 32881 und ggf. der Urinstatus (Feld 55) für die Nr. 32880 angestrichen werden. Die Anforderung von sowohl kurativen als auch präventiven Parametern erfolgt gemeinsam auf ein und demselben als präventiv gekennzeichneten Muster10A-Schein (sog. Mischfall). Das Ankreuzfeld „kurativ“ wird in diesem Fall nicht verwendet. Die Leistungen nach den neuen Gebührenordnungspositionen 32880, 32881 und 32882 können auch bei Laborarztpraxen mit dem Muster 10 angefordert werden. In diesem Fall ist die Angabe der o. g. Gebührenordnungsposition(en) notwendig. 